\title{
Transportproteine
}

\section{Regulation von ABC-Transportern durch FKBPs}

\section{MARKUS GEISLER}

DEPARTMENT BIOLOGIE, UNIVERSITÄT FRIBOURG, SCHWEIZ

The plant hormone auxin is distributed in the plant by a sophisticated network of importers and exporters, including members of the $A B C B$ subclass of ATP-binding cassette $(A B C)$ transporters. ABCB-mediated auxin transport is controlled by Twisted Dwarf 1, a member of the FK506-binding protein (FKBP) family. Here, we summarize current knowledge on $A B C$ transporter regulation by FKBPs, which seems to be conserved over kingdoms and $A B C$ subfamilies arguing for conserved mechanism of plant and mammalian post-translational transporter regulation.

DOI: $10.1007 / \mathrm{s} 12268-021-1548-\mathrm{x}$

(C) Der Autor 2021, korrigierte Publikation 2021

ATP-binding cassette(ABC)-Proteine bilden ein evolutionär altes und ubiquitäres Transportsystem [1]. Die meisten ABC-Transporter sind primäre Pumpen, die die Energie der ATP-abhängigen Hydrolyse nutzen, um die verschiedensten Substrate über zelluläre Membranen zu transportieren [2]. Die Genome von Pflanzen codieren für mehr als

- Abb. 1: Twisted Dwarf1 (TWD1) reguliert die Biogenese der Auxintransporter ABCB1 und ABCB 19. A, Genetische Deletion von TWD1 (twd 1) resultiert im twisted dwarf-Syndrom, charakterisiert durch Zwergenwuchs (unten) und einer spiralförmigen Desorientierung von Organen und epidermalem Gewebe (oben), die durch Defekte im polaren Auxintransport verursacht werden [10]. Dieser Phänotyp wird durch Verlust der Auxintransporter $A B C B 1$ und ABCB19 phänokopiert. Maßstab: $10 \mu \mathrm{m}$ (oben) und $1 \mathrm{~cm}$ (unten). Die PropidiumiodidFärbungen (rot, oben) wurden [11] entnommen. B, Genetische Deletion von TWD1 (twd 1) reduziert drastisch die Plasmamembranpräsenz von $A B C B 1$ und $A B C B 19$ in der Arabidopsis-Wurzel (obere Reihe), die im endoplasmatische Retikulum zurückgehalten werden (untere Reihe). Maßstab: 50 um.
100 ABC-Proteine (die Modellpflanze Arabi dopsis thaliana enthält etwa 130 ABC-Gene), damit sind sie in Pflanzen fast doppelt so zahlreich wie in Tieren oder Mikroorganismen [3]. Ursprünglich wurden pflanzliche ABCs als Transporter beschrieben, die an Entgiftungsprozessen beteiligt sind $[4,5]$. In den letzten Jahren wurde jedoch gezeigt, dass viele von ihnen funktionell an einer

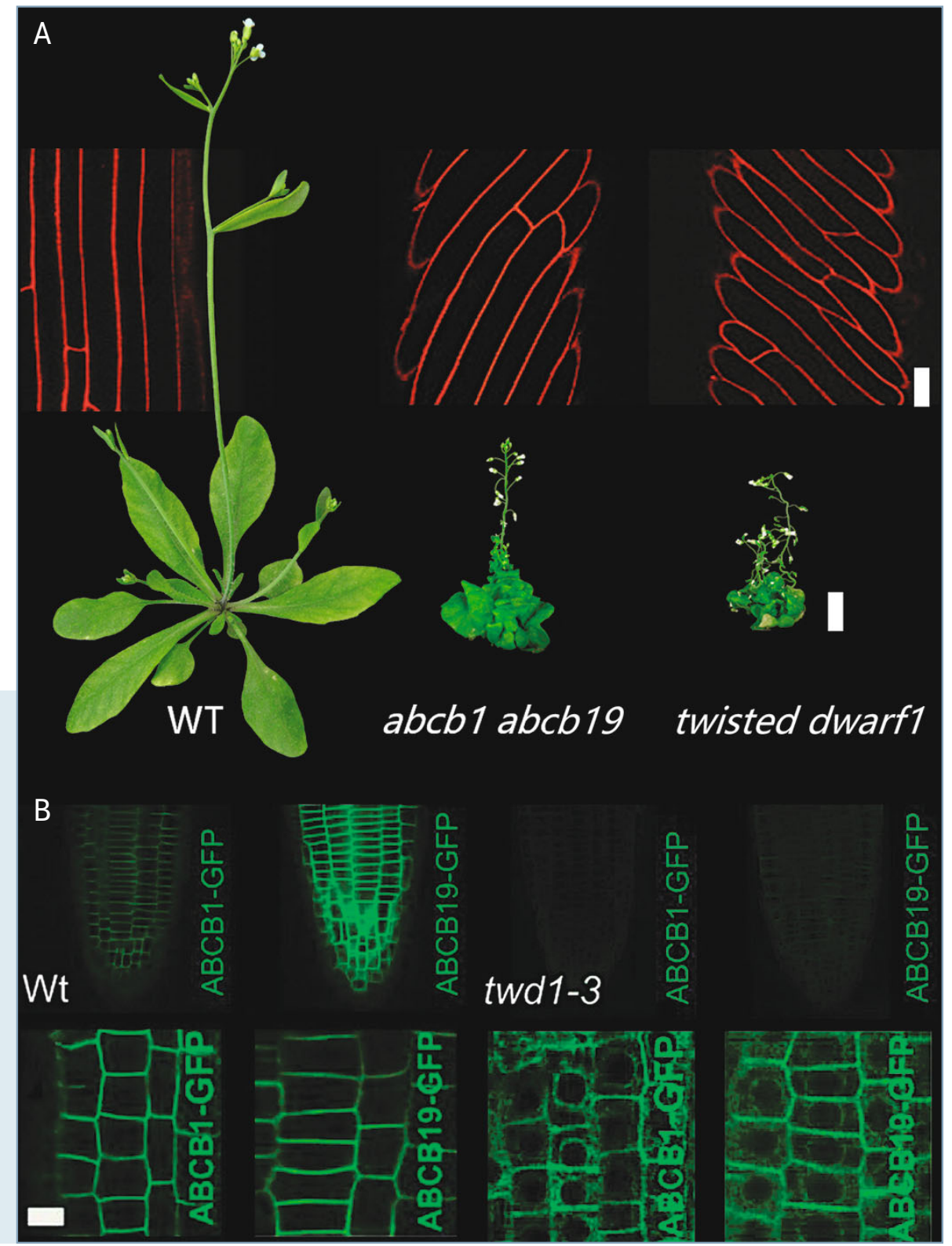



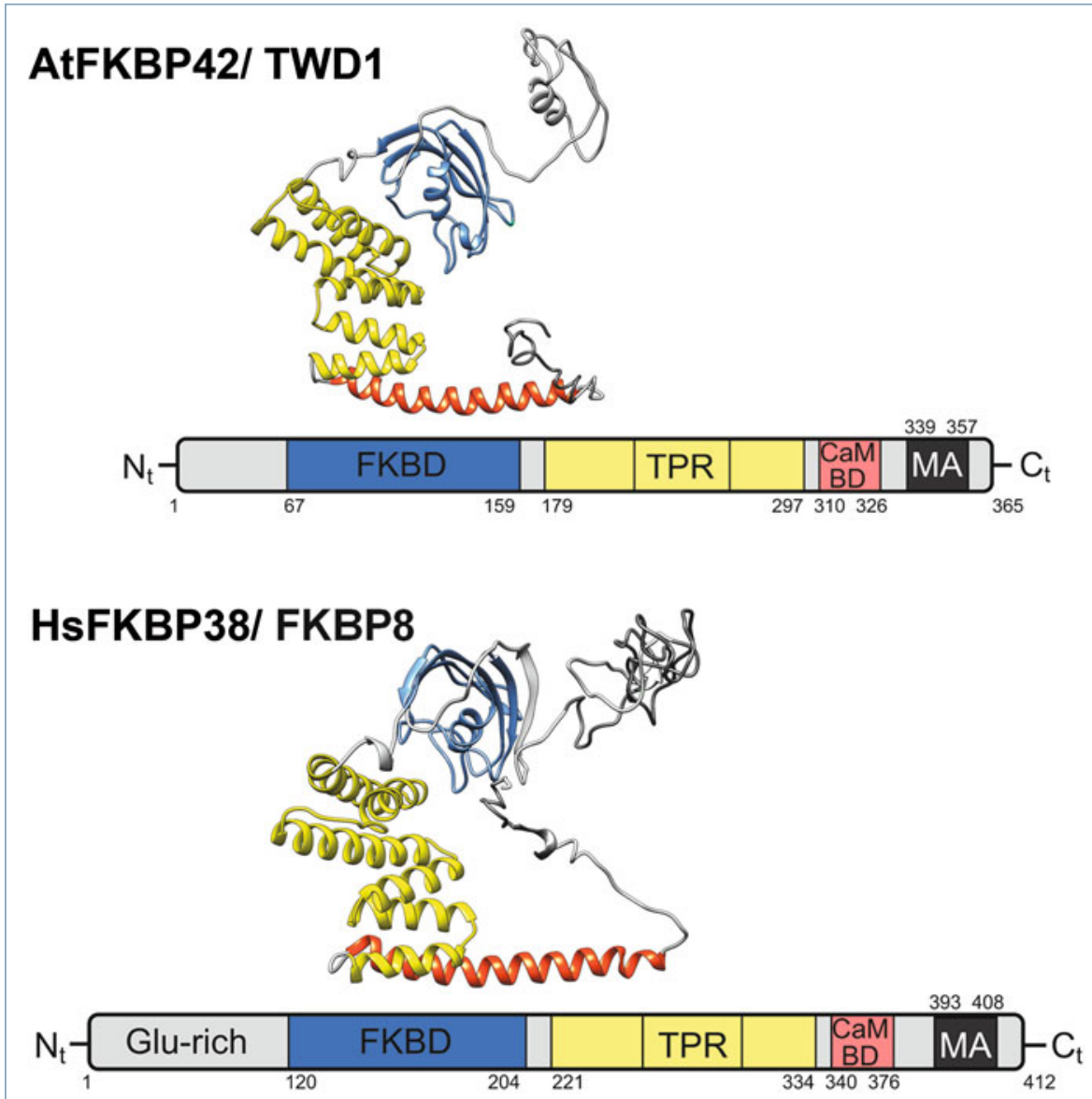

A Abb. 2: Vergleich der Molekül- und Domänenstrukturen von Arabidopsis FKBP42/TWD1 und humanem FKBP38. Die Cartoons zeigen die Konservierung der funktionellen Domänen in den beiden Multidomänen-FKBPs. Die Proteinstrukturen dienen der Illustration und wurden mit dem I-TASSER-Server (https:/ / zhanglab.ccmb.med.umich.edu/I-TASSER) generiert. Funktionelle Domänen sind gekennzeichnet: FKBD: FK506-Bindungsdomäne; TPR: Tetratricopeptid-RepeatDomäne; CaM-BD: Calmodulin-Bindungsdomäne; MA: In-Plane-Membrananker; Glu-rich: Glutamin-reiche Domäne (ERD). Aminosäuregrenzen sind angegeben. Abbildung basiert auf [14].

Vielzahl essenzieller physiologischer Prozesse beteiligt sind, wie z. B. dem Hormontransport, der Pathogenresistenz und dem abiotischen Stress [6]. Einige Mitglieder der B-TypABC-Transporter(ABCB)-Familie haben besondere Aufmerksamkeit erhalten, da für bestimmte Isoformen gezeigt wurde, dass sie am Transport des Phytohormons Auxin beteiligt sind [6].

\section{Auxin steuert die pflanzliche Entwicklung und wird von Zelle zu Zelle transportiert}

Auxin (Indolylessigsäure, IAA) spielt eine wichtige Rolle bei der Anpassung von Pflanzen an ihre Umgebung, da dieses Hormon die Orientierung und Positionierung von Organen festlegt [6]. Grundlage dafür sind lokale Auxin-Maxima, die über einen bemerkenswerten Mechanismus, den polaren Auxintransport (PAT), etabliert werden, während- dessen IAA von Zelle zu Zelle transportiert wird [7]. Dies wiederum geschieht über ein kompliziertes Netzwerk von plasmamembranständigen Auxintransportern der AUX1/ LAX(auxin resistant1/like AUX1)-, PIN(pinformed)- und eben der ABCB-Familien [8]. Letztere unterscheiden sich als primär-aktive (ATP-abhängig), energetisierte Auxinpumpen wesentlich von den erstgenannten Carriern, weil sie in der Lage sind, auch gegen steile Auxingradienten zu transportieren [8]. Während AUX1/LAX als AuxinImporter charakterisiert wurden, arbeiten PINs und ABCBs als Plasmamembran-Exporter; allerdings wurden in der Modellpflanze Arabidopsis thaliana mit ABCB4/21 auch zwei fakultative Im-/Exporter beschrieben, was man von tierischen Systemen bisher noch nicht kannte [8]. In Tumorzellen arbeiten Säuger-ABCBs übrigens als unspezifische multidrug resistance-Exporter, was bedauer- licherweise oft den Erfolg von Chemotherapien negativ beeinflusst.

\section{TWISTED DWARF1 ist ein Masterregulator der Auxin- transportierenden $\mathrm{ABCBs}$}

Die Untersuchung der post-transkriptionalen Regulation von ABC-Transportern hat sich hauptsächlich auf zwei Bereiche konzentriert: auf die Regulation der Transportaktivität (hauptsächlich durch Proteinphosphorylierung) und auf die Kontrolle der Proteinhomöostase. In letzter Zeit häufen sich allerdings die Hinweise, dass sowohl die ABCTransporter-Aktivität als auch deren Homöostase durch FK506-bindende Proteine (FKBPs) reguliert werden.

Die Arabidopsis-ABCBs waren initial als Interaktanden des FKBP42, Twisted Dwarf1 (TWD1), isoliert worden und man nahm ursprünglich an, dass TWD1 die Transportaktivität von ABCBs reguliert $[9,10]$. Heute weiß man, dass eine Subgruppe von Auxintransportierenden ABCBs in der twd1-Mutante im ER zurückgehalten wird (Abb. 1B), wo die $\mathrm{ABCBs}$ wahrscheinlich über den ERAD(endoplasmic-reticulum-associated protein degradation)-Pathway degradiert werden (Abb. 3, [11, 12]). Aktuell konnte gezeigt werden, dass TWD1 wahrscheinlich mittels seiner cis-trans-Peptidylprolylisomerase (PPIase)-Aktivität ein Prolin im C-Terminus von ABCB1 faltet [13]. Insgesamt spricht das dafür, dass TWD1 eine duale Rolle der ABCBRegulation zukommt, in der es als Chaperon die ABCB-Biogenese und als PPIase die ABCB-Aktivität kontrolliert. Die erste Funktion wird wahrscheinlich durch heat shockProteine 90 (HSP90) vermittelt, die typischerweise an TPR-Domänen von Multidomain-FKBPs binden [14]. Die zweite Funktion wird wahrscheinlich durch die PPIaseAktivität codiert, die mit der N-terminalen FK506-binding protein domain (FKBD) korreliert (Abb. 2 und 3). Als Beleg dieser beiden Konzepte zeigen Doppelmutanten von $a b c b 1$ $a b c b 19$ weitgehend überlappende Phänotypen mit twd1, einschließlich des namensgebenden Zwergwuchses und einer spiralförmigen (twisted) Desorientierung von Organen und epidermalen Geweben (Abb. 1A), die durch Defekte im PAT verursacht werden [9].

\section{Das funktionelle Ortholog von TWD1/} FKBP42, FKBP38, reguliert die Synthese und Biogenese des CFTR

FKBP38 wurde zusammen mit anderen Komponenten als Teil des Interaktoms von 
CFTR(cystic fibrosis transmembrane conductance regulator)/ABCC7, einem MRP/ ABCC-Typ-ABC-Transporter, der u. a. als pulmonaler Chloridkanal fungiert, identifiziert [14]. $\triangle$ F508-CFTR ist die häufigste Krankheitsvariante und führt zur zystischen Fibrose oder Mukoviszidose, einer autosomal-rezessiv vererbten Stoffwechselerkrankung [14].

FKBP38 besitzt insgesamt die gleiche Domänenstruktur wie FKBP42/TWD1

(Abb. 2). FKBP38-Knock-down erhöht die CFTR-Biosynthese, hemmt aber die posttranslationale Faltung, was insgesamt zu verminderter Prozessierung und Plasmamemran-Abundanz führt [32]. Im Moment wird ein Modell favorisiert, in dem FKBP38 die CFTR-Faltung während seiner Biogenese primär über seine enzymatische PPIase-Aktivität reguliert, die durch TPR-gebundenes HSP90 gehemmt wird (Abb. 3). Während sich die meisten bisherigen Studien auf die Regulation der $\triangle$ F508-CFTR-Biogenese konzentriert haben, wurde der Einfluss von FKBP38 auf die CFTR-Transportaktivität erstaunlicherweise noch nicht im Detail untersucht [14].

\section{Das regulatorische $A B C-F K B P /$ HSP90-Modul ist zwischen Tieren und Pflanzen konserviert}

Ein Vergleich zeigt, dass Auxin-transportierende ABCBs und CFTR einen evolutionär konservierten, aber komplexen Regulationsmechanismus teilen, der durch physische Interaktion mit einem FKBP-HSP90-Chaperon-Modul gewährleistet wird. In diesem sind sowohl FKBP38 als auch FKBP42/TWD1 Schlüsselregulatoren der ABCB- und CFTRBiogenese (Abb. 3), wo sie zu einem frühen Schritt der Qualitätskontrolle der frischen $\mathrm{ABCs}$ beitragen. Während für FKBP38 überzeugend nachgewiesen werden konnte, dass dieser Vorgang hauptsächlich durch die PPIase-Aktivität der FKBD erfolgt, die unter positiver und negativer Kontrolle von $\mathrm{Ca}^{2+} /$ Calmodulin bzw. TPR-gebundenem HSP90 steht, ist dies für FKBP42/TWD1 noch nicht gezeigt worden. Beide FKBPs sind aber offenbar auch für spätere Stadien des Post-ER-Traffickings zur Plasmamembran wichtig und zumindest für FKBP42/TWD1 wurde ein regulatorischer Einfluss auf die Aktivität von PMresidentem ABCB1 gezeigt [13]. Der hohe Grad der evolutionären Konservierung zwischen Pflanzen und Tieren wird auch durch eine aktuelle Studie unterstützt, die zeigt, dass der Auxintransport in Arabidopsis durch nicht-steroidale entzündungshemmende Substanzen (NSAID), wie Aspirin oder Diclofenac, über eine Bindung an TWD1 inhibiert wird [15].

Auf der Seite der ABC-Transporter ist die Konservierung eines solchen regulatorischen Moduls etwas überraschend, da ABCBs und CFTR zu unterschiedlichen ABC-Unterklassen gehören, nämlich ABCBs/PGPs bzw. ABCCs/MRPs. Dies gilt aber insbesondere angesichts der Tatsache, dass solche regulatorischen FKBP-ABC-Schaltkreise bisher nicht für nicht-Auxin-transportierende, pflanzliche ABCBs oder Säugetier-ABCBs gefunden wurden [14]. Auffallend ist deshalb, dass eben genau diese ABCB und auch CFTR unseres Wissens nach die einzigen ABC-Transporter sind, die Anionen (IAA bzw. $\mathrm{Cl}^{-}$) exportieren. Eine Analyse von FKBP42/TWD1-ABCB- und FKBP38CFTR-Sequenzpaaren könnte eine mögliche Ko-Evolution oder gemeinsame mechanistische Details dieser Interaktionen aufdecken.

Ein weiterer rätselhafter Befund ist die Relevanz der Membranverankerung von FKBP38 an der mitochondrialen und ERMembran und von FKBP42/TWD1 an der ER- und Plasmamembran. Im Allgemeinen wird angenommen, dass die Membranverankerung die Rekrutierung von cytoplasmatischen Chaperonen an Membranoberflächen ermöglicht. Angesichts der Tatsache, dass die Deletion dieser Domäne aus FKBP38/FKBP42 offenbar nur einen geringen Einfluss auf die posttranslationale Faltung der ABCs hat [4], ist die Interaktion der FKBPs mit den ABC-Klienten wahrscheinlich ausreichend.

\section{Was gilt es noch zu tun?}

Ein besseres Verständnis der Funktionalitäten von FKBP42/FKBP38 im Hinblick auf die ABC-Regulation wird durch zwei Komplikationen erschwert: Erstens enthalten beide Multidomänen-FKBPs PPIase- und Chaperon-Aktivitäten, die mit FKBP- und TPR-Domänen korrelieren, die jedoch beide zur ABC-Faltung und -Reifung beitragen können. Zweitens wirken FKBP42/TWD1 und FKBP38 im 


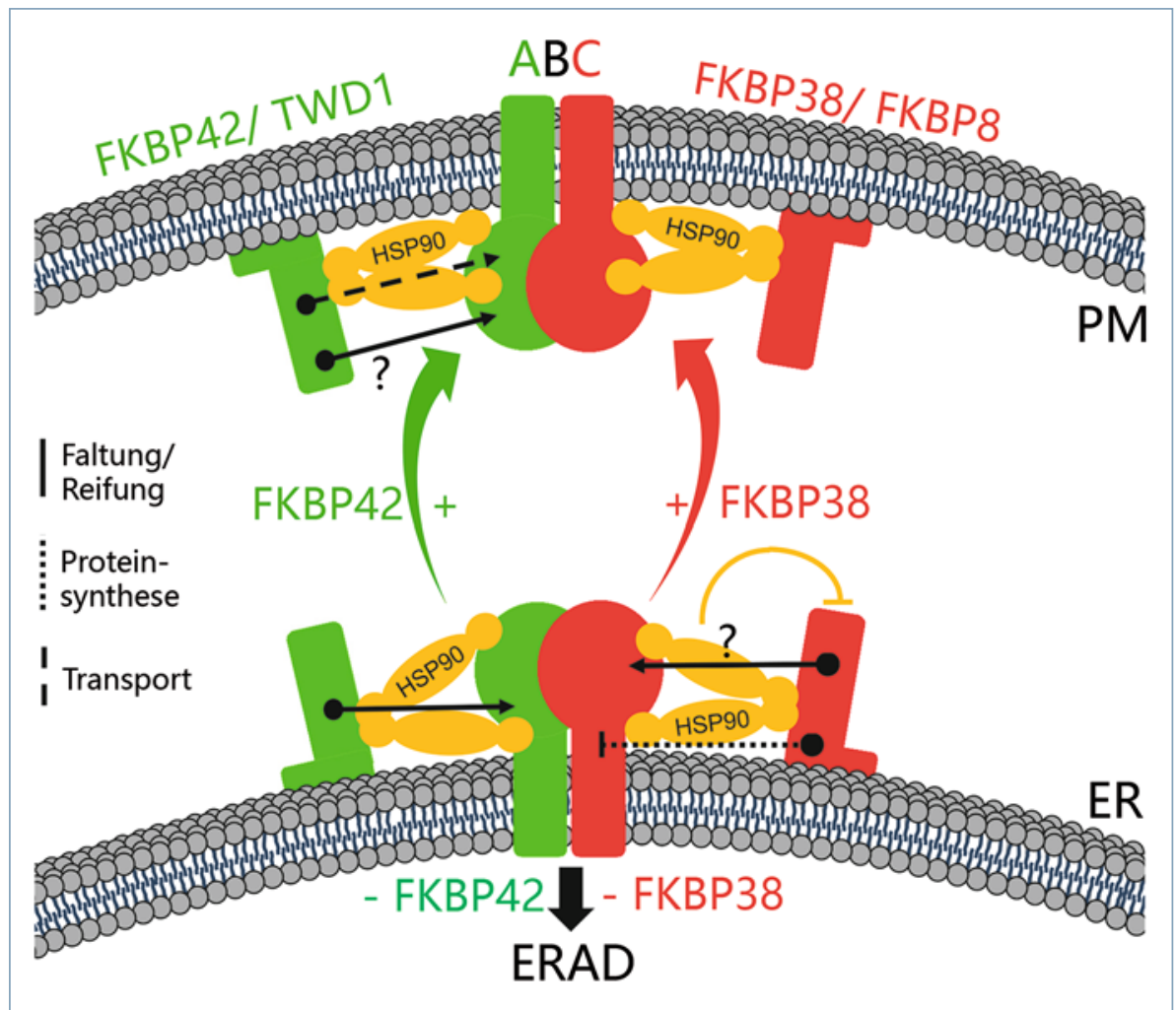

A Abb. 3: Putatives Modell der post-transkriptionalen Regulation von pflanzlichen ABCBs und humanem CFTR/ABCC7 durch FKBP42/TWD1 und FKBP38/FKBP6. Sowohl menschliches (rot) FKBP38, als auch pflanzliches FKBP42/TWD1 (grün) regulieren die Biogenese von CFTR/ABCC7 (rot) und Auxin-transportierenden ABCBs (grün) am ER. In Abwesenheit von FKBP38 und FKBP42/ TWD 1 werden die ABCs über den ERAD-Weg abgebaut. Zusätzlich sind FKBP38 und FKBP42/ TWD1 am PM-Trafficking (Pfeile) und wahrscheinlich auch an regulatorischen Ereignissen beteiligt, die den Transport oder das Kanal-Gating an der Plasmamembran verändern. Der Einfluss der FKBPs auf die Faltung/Reifung, Proteinsynthese oder den Transport ist durch durchgezogene, gepunktete oder unterbrochene Linien angegeben; positive und negative Aktionen sind durch spitze bzw. stumpfe Pfeile gekennzeichnet. Unsichere Befunde sind mit einem Fragezeichen versehen. Abbildung basiert auf [14].
[5] Di Donato M, Geisler M (2019) HSP90 and co-chaperones: a multitaskers' view on plant hormone biology. FEBS Lett 593: $1415-1430$

[6] Park J, Lee Y, Martinoia E, Geisler M (2017) Plant hormone transporters: what we know and what we would like to know. BMC Biol 15: 93

[7] Vanneste S, Friml J (2009) Auxin: a trigger for change in plant development. Cell 136: 1005-1016

[8] Geisler M, Murphy AS (2006) The ABC of auxin transport: the role of p-glycoproteins in plant development. FEBS Lett 580: 1094-1102

[9] Geisler M, Bailly A, Ivanchenko M (2016) Master and servant: regulation of auxin transporters by FKBPs and cyclophilins. Plant Sci 245: 1-10

[10] Geisler M, Kolukisaoglu HU, Bouchard R et al. (2003) TWISTED DWARF1, a unique plasma membrane-anchored immunophilin-like protein, interacts with Arabidopsis multidrug resistance-like transporters AtPGP1 and AtPGP19. Mol Biol Cell 14: 4238-4249

[11] Wu G, Otegui MS, Spalding EP (2010) The ER-localized TWD1 immunophilin is necessary for localization of multidrug resistance-like proteins required for polar auxin transport in Arabidopsis roots. Plant Cell 22: 3295-2304 [12] Wang B, Bailly A, Zwiewka M et al. (2013) Arabidopsis TWISTED DWARF1 functionally interacts with auxin exporter ABCB1 on the root plasma membrane. Plant Cell 25: 202-214 [13] Hao P, Xia J, Liu J et al. (2020) Auxin-transporting ABC transporters are defined by a conserved D/E-P motif regulated by a prolylisomerase. J Biol Chem 295: 13094-13105 [14] Geisler M, Hegedus T (2020) A twist in the ABC: regulation of $\mathrm{ABC}$ transporter trafficking and transport by FK506binding proteins. FEBS Lett, https://doi.org/10.1002/18733468.13983

[15] Tan S, Di Donato M, Glanc M et al. (2020) Non-steroidal anti-inflammatory drugs target TWISTED DWARF1-regulated actin dynamics and auxin transport-mediated plant development. Cell Rep 33: 108463

Funding note: Open access funding provided by University of Fribourg. Open Access: Dieser Artikel wird unter der Creative Commons Namensnennu 4.0 International Lizenz veröffentlicht, welche die Nutzung, Vervielfältigung, erlaubt, sofern Sie den/die ursprünglichen Autor(en) und die Quelle erdnungsgemäß $\mathrm{n}$. $\mathrm{c}$, in ordnungsgemä nennen, einen Link zur Creative Commons Lizenz beifu
angeben, ob Änderungen vorgenommen wurden. Die in diesem Artikel enthaltenen Bilder und sonstiges Drittmaterial unterliegen ebenfalls der genannten Creative Commons Lizenz, sofern sich aus der Abbildungslegende nichts anderes ergibt. Sofern das betreffende Material nicht unter der genannten Creative Commons Lizenz steht und die betreffende Handlung nich nach gesetzlichen Vorschriften erlaubt ist, ist für die oben aufgeführten Weiterverwendungen des Materials die Einwiligung des jeweiligen Rechteinhabers einzuholen. Weitere Details zur Lizenz entnehmen Sie bitte der
Gegensatz zu Single- und anderen Multidomänen-FKBPs sowohl als Chaperone als auch als Ko-Chaperone, die wiederum die Aktivität von Chaperonen beeinflussen können [5].

Angesichts der klinischen Bedeutung von CFTR ist es überraschend, dass FKBP38interagierende Oberflächen noch nicht identifiziert wurden. Während für die FKBP42/ TWD1-ABCB1-Interaktion gezeigt werden konnte, dass die FKBD von TWD1 mit der C-terminalen Nukleotid-bindenden Domäne (NBD2), die das P1008 enthält, interagiert, sind PPIase-relevante Proline im CFTR noch nicht charakterisiert worden. Allerdings scheint das P1008 aus dem Arabidopsis ABCB1 sequenziell und strukturell auch im CFTR konserviert zu sein - von daher wird es interessant sein, zu untersuchen, ob eine Mutagenese die CFTR-Transportaktivität direkt beeinflusst.

\section{Literatur}

[1] Ogasawara F, Kodan A, Ueda K (2020) ABC proteins in evolution. FEBS Lett, https://doi.org/10.1002/18733468.13945

[2] Wilkens S (2015) Structure and mechanism of ABC transporters. F1000Prime Rep 7: 14

[3] Verrier PJ, Bird D, Burla B et al. (2008) Plant ABC proteins - a unified nomenclature and updated inventory. Trends Plant Sci 13: 151-159

[4] Bailly A, Wang B, Zwiewka M et al. (2014) Expression of TWISTED DWARF1 lacking its in-plane membrane anchor leads to increased cell elongation and hypermorphic growth. Plant J 77: 108-118
Korrespondenzadresse:

Dr. Markus Geisler Department Biologie Universität Fribourg Route Albert-Gockel 10 $\mathrm{CH}-1700$ Fribourg markus.geisler@unifr.ch www3.unifr.ch/bio/en/groups/geisler

\begin{tabular}{l} 
AUTOR $\begin{array}{l}\text { Markus Geisler } \\
\text { 1986-1992 Biologiestudium an der Universität Düsseldorf mit anschliessender } \\
\text { Promotion (1996). 1996-1998 und 1999-2002 Postdoktorand an den Universitäten } \\
\text { Kopenhagen, Dänemark und Neuchâtel, Schweiz. 2002-2010 Gruppenleiter am } \\
\text { Institut für Pflanzenbiologie der Universität Zürich. Seit } 2011 \text { Lecturer an der Uni- } \\
\text { versität Fribourg. }\end{array}$ \\
\hline
\end{tabular}

